\title{
Inhomogeneous SSC model for the $\gamma$-ray production in jets of microquasars
}

\author{
Piotr Banasinski and Wlodek Bednarek \\ Department Astrophysics, University of Lodz, Poland \\ email: p.banasinski@uni.lodz.pl
}

\begin{abstract}
We present an inhomogeneous synchrotron self-Compton (SSC) model for active parts of jets in low mass microquasars. We compare results of our model computed, for typical parameters of microquasars, with the sensitivity of Fermi-LAT.
\end{abstract}

Keywords. gamma rays: theory, radiation mechanisms: nonthermal, microquasars

\section{Introduction}

Microquasars is the group of radio loud X-ray binaries with relativistic jets. These jets are expected to emit high energy (HE) radiation. In fact, observations carried out during last few years have shown that microquasars are sources of high and very high energy $\gamma$-rays. This radiation was observed only in the case of high mass microquasars where HE radiation is probably produced in the interaction of radiation from the companion star with relativistic particles in the jets. For the low mass microquasars (LM-MQ), radiation and stellar wind from a companion star is weak. However LM-MQ are expected to emit $\mathrm{HE}$ radiation produced by a relativistic plasma in a jet in the synchrotron self-Compton (SSC) process. We consider an inhomogenous SSC model for LM-MQ. The expected HE radiation is discussed in the context of sensitivities of Fermi-LAT.

\section{Model}

We consider the model of active part of jet, populated by relativistic electrons continuously accelerated along the jet. Electrons lose energy by emitting synchrotron radiation and $\mathrm{HE} \gamma$-rays in the inverse Compton process. The jet parameters change with distance from the base of a jet. To define this scenario we assume:

- The active part of the jet lays between $x_{\text {min }}=10^{3} R_{S c h}$ and $x_{\text {max }}=10^{6} R_{S c h}$.

- Magnetic field in a specific slab on the jet at the distance $x$ from the base of jet is estimated by $B(x)=B_{0} /\left(x / x_{\text {min }}\right)$, where $B_{0}$ is the field strength at the base of the jet.

- The velocity of plasma in jet $\beta_{j} c$ (where $c$ is speed of light) is independent on the distance from the base of jet.

- The maximum energy of electrons, $\gamma_{\max }$, to which they are accelerated at a specific distance along the jet, is estimated by comparing the acceleration time scale $\tau_{a c c}$ and the synchrotron cooling time scale $\tau_{\text {syn }}$. The maximum Lorentz factor of electrons at the distance $x$ is $\gamma_{\max }(x) \approx 1.1 \times 10^{8} \sqrt{\eta_{a c c} x /\left(B_{0} x_{\min }\right)}$, where $x, x_{\min }$ in $\mathrm{cm}, B_{0}$ in $\mathrm{G}$.

- The minimum energy of electron, $\gamma_{\text {min }}$, is estimated by comparing synchrotron cooling time scale, $\tau_{s y n}$, and dynamical time scale, $\tau_{d y n}=x / \beta_{j} c \mathrm{~s}$. The minimum Lorentz factor of electrons at the distance $x$ is $\gamma_{\min }(x) \approx 4.1 \times 10^{19} x /\left(x_{\min }^{2} B_{0}^{2}\right)$, where $x, x_{\min }$ in $\mathrm{cm}, B_{0}$ in $\mathrm{G}$.

- The electron injection spectrum in the slab is the power law type $d N_{e} / d E_{e} d t d x=$ $N_{e} E_{e}^{-\beta}$, where $N_{e}$ is a normalization factor and $\beta$ is spectral index. $N_{e}$ is determined 

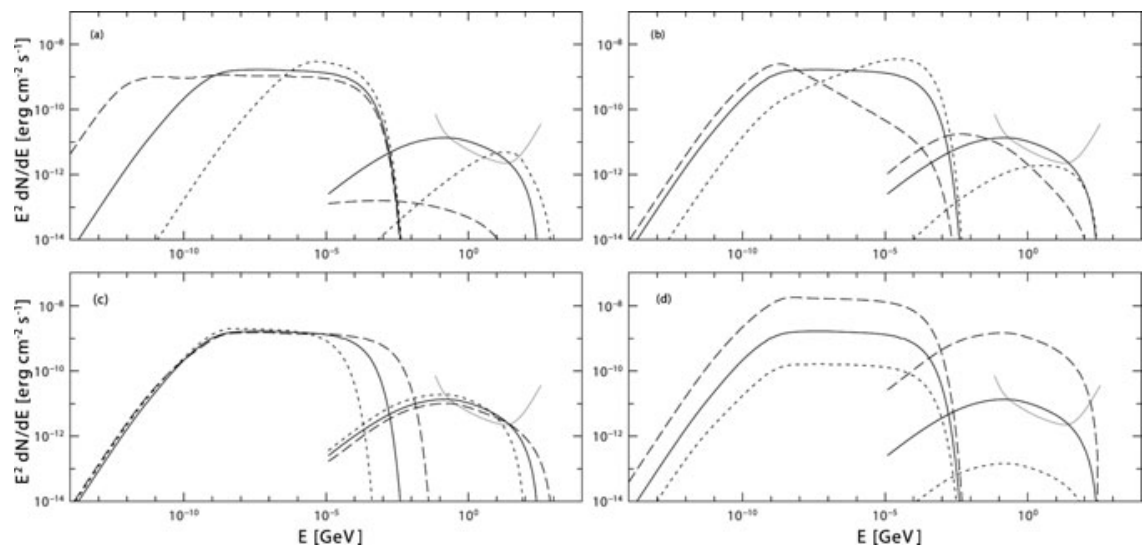

Figure 1. Synchrotron and IC SED calculated in terms of the inhomogeneous SSC model as a function of parameters describing model compared with the differential sensitivity of Fermi LAT (thick grey line) (Funk \& Hinton (2013)). (a) Dependence on the magnetic field strength at the base of the jet: $B_{0}=10^{4} \mathrm{G}$ (dotted line), $B_{0}=10^{5} \mathrm{G}$ (solid), $B_{0}=10^{6} \mathrm{G}$ (dashed). (b) Dependence on the spectral index of injected electrons: $\beta=1$ (dotted), $\beta=2$ (solid), $\beta=3$ (dashed). (c) Dependence on the acceleration coefficient of electrons: $\eta_{a c c}=10^{-4}$ (dotted), $\eta_{a c c}=10^{-3}$ (solid), $\eta_{a c c}=10^{-2}$ (dashed) (d) Dependence on the power in relativistic electrons: $\eta_{e}=10^{-4}$ (dotted), $\eta_{e}=10^{-3}$ (solid), $\eta_{e}=10^{-2}$ (dashed). Other parameters, unless otherwise noted: the black hole mass $M_{B H}=10 M_{\odot}$, the jet opening angle $\alpha=0.1 \mathrm{rad}$, distance $d=3$ $\mathrm{kpc}$, the angle between the jet axis and the line of sight $\theta=30^{\circ}$, the Lorenz factor of plasma in the jet $\Gamma_{j}=2$ and $B_{0}=10^{5} \mathrm{G}, \beta=2, \eta_{a c c}=10^{-3}, \eta_{e}=10^{-3}$.

by normalizing electron injection spectrum to the total energy in relativistic electrons, $L_{e} /\left(x_{\text {max }}-x_{\text {min }}\right)=\int_{E_{\text {min }}}^{E_{\max }} d E_{e} E_{e} d N /(d E d t d x)$, where the power in relativistic electrons is a fraction of the Eddington luminosity $L_{e}=\eta_{e} L_{e d d}$.

We take into account energy losses of electrons on the synchrotron radiation and on the inverse Compton (IC) process. The Monte Carlo method is applied in order to calculate the IC spectra in the general case.

\section{Conclusions}

We adopted a inhomogeneous SSC model to a LM-MQ in a hard state that was examined for the wide range of parameters characteristic for microquasars. We have obtained the photon spectrum indicating the fact that the MQ might be the source of HE radiation with a cutoff at $100 \mathrm{GeV}$. It is worth noticing that depending on the microquasar parameters, the level of SED $\gamma$-rays is from 1 to 3 decades lower than the level of synchrotron radiation. Some spectra can be detected by Fermi-LAT. Our conclusion agree with results for XTE J1118+480 presented in the paper of Vila \& Romero (2011). Obtained results allow us to conclude that the LM-MQ might be observed in the HE range.

\section{Acknowledgements}

This work is supported by the Polish NCN grant No. 2011/01/B/ST9/00411.

\section{References}

Funk, S. \& Hinton, J. A., for CTA Consortium 2013, Astropart. Phys., 43, 348

Vila, G. S. \& Romero, G. E. 2011, Proc. 25th Texas Symposium, arXiv:1104.2071 